\title{
ERRATUM
}

\section{Topological and Geometrical Reconstruction of Complex Objects on Irregular Isothetic Grids}

\author{
Antoine Vacavant ${ }^{1}$, David Coeurjolly ${ }^{2}$, and Laure Tougne ${ }^{1}$ \\ ${ }^{1}$ LIRIS - UMR 5205, Université Lumière Lyon 2 \\ 5, avenue Pierre Mendès-France \\ 69676 Bron cedex, France \\ ${ }^{2}$ LIRIS - UMR 5205, Université Claude Bernard Lyon 1 \\ 43, boulevard du 11 novembre 1918 \\ 69622 Villeurbanne cedex, France \\ \{antoine.vacavant, david.coeurjolly, \\ laure.tougne\}@liris.cnrs.fr
}

A. Kuba, L.G. Nyúl, and K. Palágyi (Eds.): DGCI 2006, LNCS 4245, pp. 470-481, 2006.

(C) Springer-Verlag Berlin Heidelberg 2006

\section{DOI 10.1007/11907350_58}

An error has been found in the above article.

In the original version of this paper, Fig. 2 was wrong. The correct version of figure is given below.
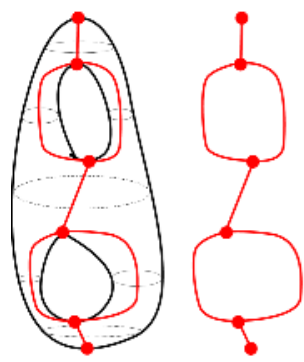

(a)
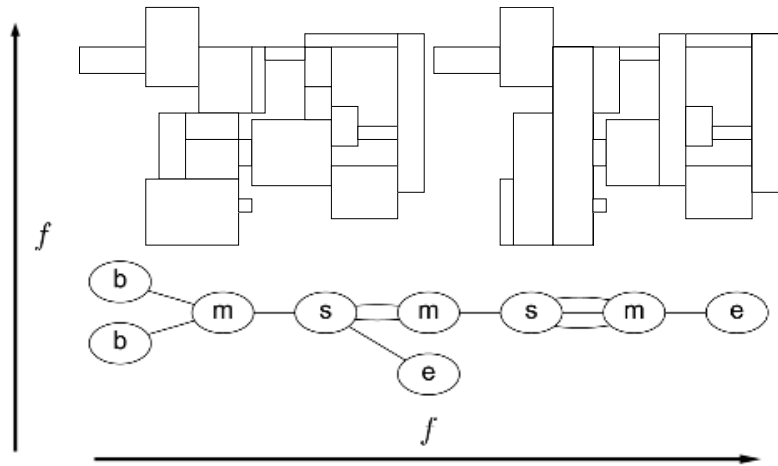

(b)

Fig. 2. (a): an example of the Reeb graph $G$ of a continuous object $E$. The nodes of $G$ represent the critical points of $f$ (maxima, minima, inflection points), and an edge is a connected component of $E$ between two critical points. (b): an example of an irregular object $E$ (left), the final recoded structure with $k$-arcs (right) and the Reeb graph associated to the height function $f$ defined on $E$ (bottom). The notations $b, e, m$ and $s$ are given at the end of this section.

The original online version for this chapter can be found at http://dx.doi.org/10.1007/11907350_40 